\title{
UNE MÉTHODE NODALE APPLIQUÉE À UN PROBLÈME DE DIFFUSION À COEFFICIENTS GÉNÉRALISÉS
}

\author{
AbdelKader LaAZizi ${ }^{1}$ et Nagib Guessous ${ }^{1}$
}

\begin{abstract}
In this paper, we consider second order neutrons diffusion problem with coefficients in $L^{\infty}(\Omega)$. Nodal method of the lowest order is applied to approximate the problem's solution. The approximation uses special basis functions [1] in which the coefficients appear. The rate of convergence obtained is $O\left(h^{2}\right)$ in $L^{2}(\Omega)$, with a free rectangular triangulation.

Résumé. Dans ce travail on considère un problème de diffusion des neutrons (à un groupe) dans un domaine $\Omega$ de $\mathbb{R}^{2}$, dont les coefficients sont dans $L^{\infty}(\Omega)$. Une méthode nodale d'ordre zéro appliquée à ce type de problèmes mène à une erreur d'approximation d'ordre $O\left(h^{2}\right)$ dans $L^{2}(\Omega)$, en utilisant des fonctions d'approximation de type spéciales [1] et dans lesquelles apparaissent les coefficients de diffusion, tout en adoptant une triangulation libre du domaine par des rectangles.
\end{abstract}

AMS Subject Classification. 65N30.

Reçu : 21 avril 1998. Révisé : 14 décembre 1998.

\section{INTRODUCTION}

Les méthodes nodales ont été utilisées pour la première fois dans les années 70 dans le calcul numérique des réacteurs nucléaires [11], dès lors elles ont connu un grand succès dans ce domaine grâce à leur efficacité et leur rapidité qui proviennent du fait que :

1. ces méthodes partagent avec les méthodes des éléments finis l'approximation de la solution par des fonctions polynômiales par morceaux (parfois ces méthodes utilisent une approximation quasi-polynômiale où en plus des fonctions polynômes apparaissent des fonctions exponentielles, c'est le cas des méthodes nodales analytiques [6]); 2. avec les méthodes de différences finies, elles ont en commun le fait que le système algébrique final possède une matrice creuse et bien structurée sur des domaines réguliers de type réunion de rectangles.

Étant donné l'ouvert $\Omega=]-1,+1\left[^{2}\right.$ de $\mathbb{R}^{2}$, de variable spatiale $x=\left(x_{1}, x_{2}\right)$, on considère le problème suivant :

$$
\begin{cases}L u=-\operatorname{div}\left(A\left(x_{1}, x_{2}\right) \nabla u\right)+g\left(x_{1}, x_{2}\right) u=f & \text { dans } \Omega \\ u=0 & \text { sur } \partial \Omega\end{cases}
$$

où $\partial \Omega=\bar{\Omega}-\Omega$ désigne la frontière de $\Omega$, et

$$
A\left(x_{1}, x_{2}\right)=\left[\begin{array}{cc}
a_{1}\left(x_{1}\right) b_{1}\left(x_{2}\right) & 0 \\
0 & a_{2}\left(x_{1}\right) b_{2}\left(x_{2}\right)
\end{array}\right] .
$$

Keywords and phrases. Nodal methods, diffusion problem, rough coefficients.

${ }^{1}$ Faculté des Sciences Dhar-Mehraz, Fès, U.F.R. de Mathématiques Appliquées, Laboratoire d'Analyse Numérique, B.P. 1796 Fès-Atlas, Maroc. e-mail: laazizi@yahoo.com 
Avec l'existence de deux constantes : $c_{1}, c_{2}>0$ telles que :

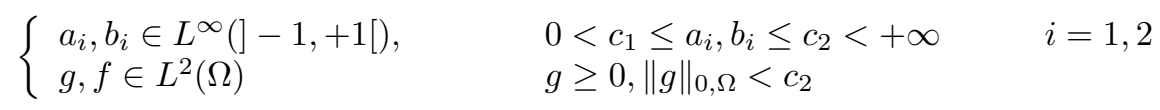

on pose $A_{11}(x)=a_{1}\left(x_{1}\right) b_{1}\left(x_{2}\right), A_{22}(x)=a_{2}\left(x_{1}\right) b_{2}\left(x_{2}\right)$. $A(x)$ représente le terme de diffusion et $g$ celui d'absorption.

Le but de ce travail est d'appliquer une méthode des éléments finis de type nodale d'ordre zéro pour approcher la solution $u$ du problème (1.1).

Dans le paragraphe suivant on va rappeler les principes de base des méthodes nodales standards i.e. quand les coefficients $A$ et $g$ sont constants par morceaux sur une triangulation rectangulaire $T_{h}$ de $\Omega$, ainsi que les critères de convergence de ces méthodes, dans le paragraphe 3 on va énoncer la généralisation du théorème de Bernstein [3] avec les conditions suffisantes d'existence et d'unicité de la solution du problème (1.1), le paragraphe 4 sera réservé à l'exposé de la méthode nodale à coefficients généralisés $\left(\left(A_{i i}\right)_{1 \leq i \leq 2} \in L^{\infty}\right)$, voir $(1.2)$, ainsi que la convergence de la solution approchée. Les résultats numériques sont donnés dans le paragraphe 5. Ce travail rejoint celui de $[1,2,9]$.

\section{Principes Des mÉthodes nOdAles StANDARDS D'ORDRE $k$}

Durant toute l'étude on aura besoin des notations suivantes :

$$
H^{k}(\Omega)=\left\{u \in L^{2}(\Omega): D^{\alpha} u \in L^{2}(\Omega),|\alpha| \leq k\right\}
$$

où $D^{\alpha}$ est la dérivée (au sens des distributions) d'ordre $\alpha$ de la fonction $u ; H^{k}(\Omega)$ sera muni de la norme et de la semi-norme suivantes :

$$
\|u\|_{k, \Omega}^{2}=\sum_{|\alpha| \leq k}\left\|D^{\alpha} u\right\|_{0, \Omega}^{2} ; \quad|u|_{k, \Omega}^{2}=\sum_{|\alpha|=k}\left\|D^{\alpha} u\right\|_{0, \Omega}^{2}
$$

où $\|u\|_{0, \Omega}$ est la norme dans $L^{2}(\Omega)$ : l'espace des fonctions définies sur $\Omega$, mesurables et de carrés sommables avec : $\|u\|_{0, \Omega}^{2}=\int_{\Omega} u^{2} \mathrm{~d} x, \forall u \in L^{2}(\Omega)$.

Considérons le problème suivant :

$$
\begin{cases}\mathcal{L}_{1} u=-\operatorname{div}(D \nabla u)+C u=f & \text { dans } \Omega \\ u=0 & \text { sur } \partial \Omega\end{cases}
$$

Le domaine $\Omega=]-1,+1\left[{ }^{2}\right.$ est partagé en des rectangles $\Omega_{i}$ tels que $: \bar{\Omega}=\cup \bar{\Omega}_{i}$, on désigne par $h_{i}$ (resp. $\rho_{i}$ ) le diamètre (resp. la rondeur) de $\Omega_{i}$ et $h=\max h_{i}$, les domaines $\Omega_{i}$ sont pris tels que $: h_{i} / \rho_{i} \leq \sigma, \sigma>0$, i.e. de telle sorte que la triangulation $T_{h}$ de $\Omega$ soit régulière ([5], p. 131).

Le domaine $\Omega$ est partagé de telle sorte que les coefficients $D$ et $C$ du problème (2.1) soient constants sur chaque $\Omega_{i}$ afin d'avoir le maximum de régularité de $u$ sur $\Omega_{i}$. Dans le langage du neutronique, les quantités $u$ et $\vec{p}=-D . \nabla u$ désignent respectivement le flux et le courant des neutrons ; on pose :

$$
P_{k}=\left\{x_{1}^{a} x_{2}^{b}: 0 \leq a+b \leq k\right\} \quad Q_{k, l}=\left\{x_{1}^{r} x_{2}^{s}: 0 \leq r \leq k, 0 \leq s \leq l\right\} .
$$

On désigne par $p_{k}$ le polynôme de Legendre de degré $k$ défini pour tout $t \in[-1,+1]$ par :

$$
p_{k}(t)=\frac{1}{2^{k} k !} \frac{\mathrm{d}^{k}}{\mathrm{~d} t^{k}}\left(t^{2}-1\right)^{k} .
$$

Ces polynômes vérifient

$$
p_{k}(+1)=+1, \quad p_{k}(-1)=(-1)^{k} \quad \forall k \in \mathbb{N}
$$




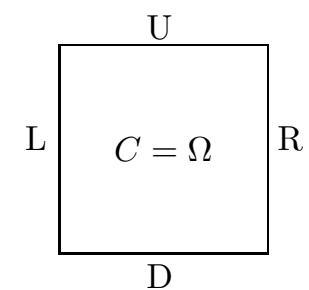

FiguRe 1.

et la relation d'orthogonalité suivante :

$$
\frac{1}{N_{k}} \int_{-1}^{1} p_{k}(t) p_{l}(t) \mathrm{d} t= \begin{cases}1 & \text { si } k=l \\ 0 & \text { sinon. }\end{cases}
$$

Avec $N_{k}=2 /(2 k+1)$; on convient d'écrire $p_{k l}\left(x_{1}, x_{2}\right)=p_{k}\left(x_{1}\right) p_{l}\left(x_{2}\right)$ : l'écriture condensée de ces polynômes.

Pour toute fonction $u$ définie sur $[-1,+1]^{2}$, on introduit les fonctionnelles linéaires : $\left(m_{C}^{i j}(u), m_{L}^{i}(u), m_{R}^{i}(u), m_{D}^{i}(u), m_{U}^{i}(u)\right)$ associées au domaine $]-1,+1\left[{ }^{2}\right.$ et définies par :

$$
m_{C}^{i j}(u)=\int_{C} p_{i j}\left(x_{1}, x_{2}\right) u\left(x_{1}, x_{2}\right) \mathrm{d} x / N_{i j}, \quad i, j=0 \ldots k
$$

avec $N_{i j}=N_{i} N_{j}$, et sur les faces $L, R, D, U, C$ (voir Fig. 1) :

$$
\left\{\begin{array}{ll}
m_{L}^{i}(u)=\int_{-1}^{+1} p_{i}\left(x_{2}\right) u\left(-1, x_{2}\right) \mathrm{d} x_{2} / N_{i} & \\
m_{R}^{i}(u) & =\int_{-1}^{+1} p_{i}\left(x_{2}\right) u\left(+1, x_{2}\right) \mathrm{d} x_{2} / N_{i} \\
m_{U}^{i}(u) & =\int_{-1}^{+1} p_{i}\left(x_{1}\right) u\left(x_{1},+1\right) \mathrm{d} x_{1} / N_{i} \\
m_{D}^{i}(u) & =\int_{-1}^{+1} p_{i}\left(x_{1}\right) u\left(x_{1},-1\right) \mathrm{d} x_{1} / N_{i}
\end{array} \quad i=0, \ldots, k\right.
$$

où $m_{E}^{i}(u)$ est le moment d'ordre $i$ de la fonction $u$ sur la face $E \in\{L, R, U, D\}$, quand $i=j=0$ on se contente d'écrire $m_{C}, m_{L}, m_{R}, m_{U}$ et $m_{D}$.

Les fonctions de base correspondantes à $m_{C}^{i j} \quad\left(\right.$ resp. à $\left.m_{L}^{i}, m_{R}^{i}, m_{D}^{i}, m_{U}^{i}\right)$ sont $u_{C}^{i j} \quad\left(\right.$ resp. $\left.u_{L}^{i}, u_{R}^{i}, u_{D}^{i}, u_{U}^{i}\right)$ définies (voir [7]) par :

$$
\left\{\begin{array}{rl}
u_{C}^{i j}\left(x_{1}, x_{2}\right) & =p_{i j}\left(x_{1}, x_{2}\right)-p_{k+l(i), j}\left(x_{1}, x_{2}\right)-p_{i, k+l(j)}\left(x_{1}, x_{2}\right) \\
u_{L}^{i}\left(x_{1}, x_{2}\right) & =\frac{1}{2}(-1)^{k+1}\left(p_{k+1}\left(x_{1}\right)-p_{k+2}\left(x_{1}\right)\right) p_{i}\left(x_{2}\right) \\
u_{U}^{i}\left(x_{1}, x_{2}\right) & =\frac{1}{2}\left(p_{k+1}\left(x_{2}\right)+p_{k+2}\left(x_{2}\right)\right) p_{i}\left(x_{1}\right) \\
u_{R}^{i}\left(x_{1}, x_{2}\right) & =\frac{1}{2}\left(p_{k+1}\left(x_{1}\right)+p_{k+2}\left(x_{1}\right)\right) p_{i}\left(x_{2}\right) \\
u_{D}^{i}\left(x_{1}, x_{2}\right) & =\frac{1}{2}(-1)^{k+1}\left(p_{k+1}\left(x_{2}\right)-p_{k+2}\left(x_{2}\right)\right) p_{i}\left(x_{1}\right)
\end{array} \quad i=0, \ldots, k\right.
$$

où $l(n)=1$ ou 2 tel que $k+l(n)$ et $n$ ont même parité, de même quand $i=j=0$ on écrit $u_{L}, u_{U}, u_{R}, u_{D}$ et $u_{C}$. 
La méthode nodale appliquée au problème (2.1) consiste à chercher une approximation $u_{h}$ de $u$ dans un sous espace de dimension finie :

$$
\begin{aligned}
\mathcal{U}_{h}=\{ & v_{h} \in L^{2}(\Omega): \forall \Omega_{i} \in T_{h}, v_{h} / \Omega_{i} \in P_{k}\left(\Omega_{i}\right), \\
& \left.m_{E}^{i}\left(v_{h}\right) \text { est continu à travers les interfaces des } \Omega_{i}, m_{E}^{i}(v)=0 \text { si } E \subset \partial \Omega\right\} .
\end{aligned}
$$

L'approximation $u_{h}$ est donnée en terme des moments $m_{C}^{i j}$ sur $\Omega_{i}$ et $m_{E}^{i}(E \in\{L, R, U, D\})$ sur $\partial \Omega_{i}$, sa forme est :

$$
u_{h}\left(x_{1}, x_{2}\right)=\sum_{i, j=0}^{k} m_{C}^{i j} u_{C}^{i j}+\sum_{i=0}^{k} m_{E}^{i} u_{E}^{i}
$$

Ceci car chaque $\Omega_{i}$ peut se transformer en $]-1,+1\left[2\right.$ grâce a une application affine diagonale. Les éléments $v_{h}$ de $\mathcal{U}_{h}$ auront une continuité juste en terme des moments $m_{E}^{i}$, mais ils ne sont pas nécessairement continus sur les interfaces des $\Omega_{i}$ ce qui nous amène à une approximation non-conforme [5] i.e. $\mathcal{U}_{h} \not \subset H_{0}^{1}(\Omega)$.

Remarque 1. En présence des discontinuités des coefficients à travers les interfaces des $\Omega_{i}$ la solution $u$ présente des singularités en quelques points (voir[8]) et sa forme générale est : $u(x)=u_{r}(x)+\sum_{s=1}^{M} u_{s}(x)$; où $M$ est le nombre des points (noeuds) internes à $\Omega$, et $u_{r}$ est la partie régulière de $u$ i.e. $u_{r} \in H_{0}^{1}(\Omega) \cap H^{2}(\Omega)$, alors que $u_{s}$ est la partie singulière associée au points singuliers : $u_{s} \notin H^{2}(\Omega)$ c'est-à-dire $u \notin H^{2}(\Omega)$.

Avec ces fonctions de base définies en (2.3) si on pose : $D_{k}=\left\{m_{C}^{i j}, m_{L}^{i}, m_{R}^{i}, m_{D}^{i}, m_{U}^{i}\right\}$, et $S_{k}=Q_{k+2, k} \cup$ $Q_{k, k+2}$, alors $\left(u_{C}^{i j}, u_{E}^{i}\right) \in S_{k}^{2}, \quad \forall E \in\{L, R, D, U\}$; et on a le résultat suivant [6] :

Théorème 1. $S_{k}$ est $D_{k}$-unisolvant, et si u est assez régulière alors il existe une constante c indépendante de $h$ telle que:

$$
\left\|u-u_{h}\right\|_{0, \Omega} \leq c h^{k+2}|u|_{k+2, \Omega} .
$$

\section{Localisation de la SOlution DE L'opérateur $L$}

Considérons la formulation variationnelle du problème (1.1) :

$$
\left\{\begin{array}{l}
u \in H_{0}^{1}(\Omega) \\
\int_{\Omega} \sum_{i=1}^{2} a_{i}\left(x_{1}\right) b_{i}\left(x_{2}\right) \frac{\partial u}{\partial x_{i}} \frac{\partial v}{\partial x_{i}}+g\left(x_{1}, x_{2}\right) u v \mathrm{~d} x=\int_{\Omega} f v \mathrm{~d} x, \forall v \in H_{0}^{1}(\Omega) .
\end{array}\right.
$$

On pose :

$$
\left\{\begin{aligned}
a(u, v) & =\int_{\Omega} \sum_{i=1}^{2} a_{i}\left(x_{1}\right) b_{i}\left(x_{2}\right) \frac{\partial u}{\partial x_{i}} \frac{\partial v}{\partial x_{i}}+g\left(x_{1}, x_{2}\right) u v \mathrm{~d} x \\
f(v) & =\int_{\Omega} f v \mathrm{~d} x
\end{aligned}\right.
$$

D'après la théorie de Lax-Milgram (cf. [5] p. 29), le problème (3.1) admet une solution unique dans $H_{0}^{1}(\Omega)$, et vu la non régularité des coefficients, la solution $u \notin H^{2}(\Omega)$; or l'application de la théorie des méthodes nodales exige que $u$ soit dans $H^{k+2}(\Omega)$ pour avoir une approximation d'ordre $O\left(h^{k+2}\right)$ dans $L^{2}(\Omega)$, pour cela nous allons nous baser sur les résultats de [1], là où une méthode spéciale d'éléments finis est construite pour 
le problème (1.1) sans le terme d'absorption $g$. L'étude sera basée sur le théorème suivant qui généralise le théorème de Bernstein [3], et qui peut être déduit facilement d'un résultat de [10] (p. 237) :

Théorème 2. Étant donné $\Omega=]-1,+1\left[{ }^{2}\right.$ et l'opérateur $L_{2}$ tel que :

$$
\begin{cases}L_{2} u=-\sum_{i, j=1}^{2} a_{i j}(x) \frac{\partial^{2} u}{\partial x_{i} \partial x_{j}}+g u=f & \text { dans } \Omega \\ u=0 & \text { sur } \partial \Omega\end{cases}
$$

avec l'existence de $\alpha_{1}, \alpha_{2}$ deux constantes strictement positives telles que:

$$
\alpha_{1} \sum_{i=1}^{2} \xi_{i}^{2} \leq \sum_{i, j=1}^{2} a_{i j} \xi_{i} \xi_{j} \leq \alpha_{2} \sum_{i=1}^{2} \xi_{i}^{2}, f \in L^{2}(\Omega), 0 \leq g, \text { et }\|g\|_{0, \Omega} \leq \alpha_{2}
$$

alors le problème (3.3) admet une solution unique dans $H^{2}(\Omega) \cap H_{0}^{1}(\Omega), \forall f \in L^{2}(\Omega)$ si et seulement si le théorème d'unicité est vérifié.

On va ramener le problème (1.1) à un problème de type (3.3), pour cela on pose :

$$
\hat{A}\left(x_{1}\right)=\int_{-1}^{x_{1}} \frac{1}{a_{1}(t)} \mathrm{d} t, \quad \hat{B}\left(x_{2}\right)=\int_{-1}^{x_{2}} \frac{1}{b_{2}(t)} \mathrm{d} t, \quad \forall\left(x_{1}, x_{2}\right) \in[-1,+1]^{2} .
$$

On pose aussi la transformation suivante :

$$
\left\{\begin{aligned}
t_{1}\left(x_{1}\right) & =\left(\frac{2}{\hat{A}(1)} \hat{A}\left(x_{1}\right)\right)-1 \\
t_{2}\left(x_{2}\right) & =\left(\frac{2}{\hat{B}(1)} \hat{B}\left(x_{2}\right)\right)-1
\end{aligned}\right.
$$

On va noter $\left(t_{1}, t_{2}\right)$ au lieu de $\left(t_{1}\left(x_{1}\right), t_{2}\left(x_{2}\right)\right)$ pour alléger l'écriture.

L'application (3.4) transforme $\Omega=]-1,+1\left[{ }^{2}\right.$ du plan $\left(x_{1}, x_{2}\right)$ en $\left.\hat{\Omega}=\right]-1,+1\left[^{2}\right.$ du plan $\left(t_{1}, t_{2}\right)$. On définit l'espace $H^{L}(\Omega)$ : espace introduit par Babûska-Osborn dans [2] par :

$$
H^{L}(\Omega)=\left\{u \in H^{1}(\Omega): a_{1}\left(x_{1}\right) \frac{\partial u}{\partial x_{1}}, b_{2}\left(x_{2}\right) \frac{\partial u}{\partial x_{2}} \in H^{1}(\Omega)\right\}
$$

qu'on munit de la norme suivante : $\|u\|_{L, \Omega}^{2}=\|u\|_{1, \Omega}^{2}+|u|_{L, \Omega}^{2}$ avec

$$
|u|_{L, \Omega}^{2}=\int_{\Omega}\left[\frac{a_{1}}{b_{2}}\left|\frac{\partial}{\partial x_{1}}\left(a_{1} \partial u / \partial x_{1}\right)\right|^{2}+\frac{a_{1}}{b_{2}}\left|\frac{\partial}{\partial x_{1}}\left(b_{2} \partial u / \partial x_{2}\right)\right|^{2}+\frac{b_{2}}{a_{1}}\left|\frac{\partial}{\partial x_{2}}\left(b_{2} \partial u / \partial x_{2}\right)\right|^{2}\right] \mathrm{d} x
$$

On pose :

$$
\hat{u}\left(t_{1}\left(x_{1}\right), t_{2}\left(x_{2}\right)\right)=u\left(x_{1}, x_{2}\right), \forall x_{1}, x_{2} \in \Omega
$$

et on remarque que :

$$
\hat{u} \in H^{2}(\hat{\Omega}) \Leftrightarrow u \in H^{L}(\Omega) .
$$




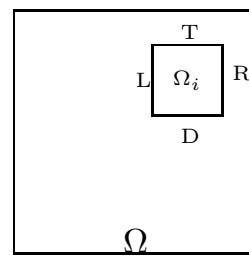

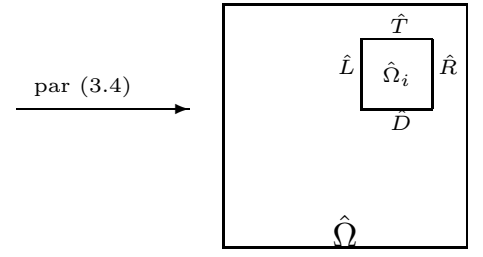

FiguRE 2.

En utilisant la transformation (3.4), la formulation variationnelle (3.1) du problème (1.1) devient :

$$
\left\{\begin{array}{l}
\hat{u} \in H_{0}^{1}(\hat{\Omega}) \\
\int_{\hat{\Omega}} \hat{b}_{1} \hat{b}_{2} \frac{\partial \hat{u}}{\partial t_{1}} \frac{\partial \hat{v}}{\partial t_{1}}+\hat{a}_{1} \hat{a}_{2} \frac{\partial \hat{u}}{\partial t_{2}} \frac{\partial \hat{v}}{\partial t_{2}}+\hat{a}_{1} \hat{b}_{2} \hat{g}\left(t_{1}, t_{2}\right) \hat{u} \hat{v} \mathrm{~d} t=\int_{\hat{\Omega}} \hat{f} \hat{v} \hat{a}_{1} \hat{b}_{2} \mathrm{~d} t, \forall \hat{v} \in H_{0}^{1}(\hat{\Omega}) .
\end{array}\right.
$$

Les $\hat{a}_{i}$ sont des fonctions de $t_{1}$, les $\hat{b}_{i}$ sont des fonctions de $t_{2}$ et on a :

$$
0<c_{1} \leq \hat{a}_{i}, \hat{b}_{i} \leq c_{2}<\infty, \quad \mathrm{d} t=\mathrm{d} t_{1} \mathrm{~d} t_{2}
$$

On remarque que (3.8) est la formulation variationnelle de :

$$
\begin{cases}-\hat{b}_{1} \hat{b}_{2} \frac{\partial^{2} \hat{u}}{\partial t_{1}^{2}}-\hat{a}_{1} \hat{a}_{2} \frac{\partial^{2} \hat{u}}{\partial t_{2}^{2}}+\hat{g} \hat{a}_{1} \hat{b}_{2} \hat{u}=\hat{f} \hat{a}_{1} \hat{b}_{2}, & \text { dans } \hat{\Omega} \\ \hat{u}=0 & \text { sur } \partial \hat{\Omega}\end{cases}
$$

Et on a le résultat suivant :

Lemme 1. Si le problème (3.8) admet une solution dans $H_{0}^{1}(\hat{\Omega}) \cap H^{2}(\hat{\Omega})$, alors elle est unique.

En effet : soient $\hat{u}_{1}, \hat{u}_{2}$ deux solutions du problème (3.8) alors $\hat{u}_{1}-\hat{u}_{2}$ vérifie :

$$
\int_{\hat{\Omega}}\left[\hat{b}_{1} \hat{b}_{2}\left|\frac{\partial}{\partial t_{1}}\left(\hat{u}_{1}-\hat{u}_{2}\right)\right|^{2}+\hat{a}_{1} \hat{a}_{2}\left|\frac{\partial}{\partial t_{2}}\left(\hat{u}_{1}-\hat{u}_{2}\right)\right|^{2}+\hat{g} \hat{a}_{1} \hat{b}_{2}\left|\hat{u}_{1}-\hat{u}_{2}\right|^{2}\right] \mathrm{d} t=0
$$

c'est-à-dire que $\hat{u}_{1}-\hat{u}_{2}=$ const. sur $\hat{\Omega}$ qui est un connexe, or sur $\partial \Omega$ on a $\hat{u}_{1}-\hat{u}_{2}=0$ donc $\hat{u}_{1}=\hat{u}_{2}$ dans $H_{0}^{1}(\hat{\Omega})$ et on a le résultat suivant :

Théorème 3. Pour tout $f \in L^{2}(\Omega)$ le problème (1.1) admet une solution unique dans $H^{L}(\Omega) \cap H_{0}^{1}(\Omega)$ et il existe une constante $c>0$ telle que :

$$
\|u\|_{L, \Omega} \leq c\|f\|_{0, \Omega}
$$

En effet, il suffit d'adopter la démonstration du théorème (2.2) de [1] en utilisant la transformation (3.4), le théorème 2 et le lemme précédent.

\section{LA MÉTHODE NODALE À COEFFICIENTS GÉNÉRALISÉS}

Le domaine $\Omega=]-1,+1\left[{ }^{2}\right.$ est partagé en des rectangles $\Omega_{i}$ de frontière $(L \cup R \cup U \cup D), \Omega_{i}$ a pour image, par la transformation (3.4), l'ouvert $\hat{\Omega}_{i}$ de $]-1,+1\left[^{2}\right.$ dans le plan $\left(t_{1}, t_{2}\right)$, de frontière $(\hat{L} \cup \hat{R} \cup \hat{U} \cup \hat{D})$ (voir Fig. 2), le domaine $\hat{\Omega}$ est tel que $: \overline{\hat{\Omega}}=\cup \overline{\hat{\Omega}}_{i}$. 
Si $\hat{h}_{i}$ (resp. $\left.\hat{\rho}_{i}\right)$ désigne le diamètre (resp. la rondeur ) de $\hat{\Omega}_{i}$ on a :

$$
\hat{h}_{i} \leq \max \left(\frac{1}{c_{1}}, 1\right) h_{i} \leq \max \left(\frac{1}{c_{1}}, 1\right) h, \quad \rho_{i} \leq c \hat{\rho}_{i}
$$

de telle sorte qu'on obtient encore une triangulation régulière de $\hat{\Omega}$.

Pour tout $\Omega_{i} \in T_{h}$, on pose :

$$
P\left(\Omega_{i}\right)=\left\{1, \hat{A}\left(x_{1}\right),\left(\hat{A}\left(x_{1}\right)\right)^{2}, \hat{B}\left(x_{2}\right),\left(\hat{B}\left(x_{2}\right)\right)^{2}\right\}
$$

c'est-à-dire l'espace engendré par :

$$
\left\{1, \int_{-1}^{x_{1}} \frac{1}{a_{1}(t)} \mathrm{d} t,\left(\int_{-1}^{x_{1}} \frac{1}{a_{1}(t)} \mathrm{d} t\right)^{2}, \int_{-1}^{x_{2}} \frac{1}{b_{2}(t)} \mathrm{d} t,\left(\int_{-1}^{x_{2}} \frac{1}{b_{2}(t)} \mathrm{d} t\right)^{2}\right\}
$$

Soit $D$ l'ensemble des degrés de liberté défini par :

$$
D=\left\{m_{L}^{b_{2}}, m_{R}^{b_{2}}, m_{U}^{a_{1}}, m_{D}^{a_{1}}, m_{C}^{a_{1} b_{2}}\right\}
$$

Avec

$$
\left\{\begin{array}{l}
m_{L}^{b_{2}}(u)=\frac{1}{\hat{B}(1)} \int_{-1}^{+1} \frac{1}{b_{2}\left(x_{2}\right)} u\left(-1, x_{2}\right) \mathrm{d} x_{2} \\
m_{R}^{b_{2}}(u)=\frac{1}{\hat{B}(1)} \int_{-1}^{+1} \frac{1}{b_{2}\left(x_{2}\right)} u\left(+1, x_{2}\right) \mathrm{d} x_{2} \\
m_{D}^{a_{1}}(u)=\frac{1}{\hat{A}(1)} \int_{-1}^{+1} \frac{1}{a_{1}\left(x_{1}\right)} u\left(x_{1},-1\right) \mathrm{d} x_{1} \\
m_{U}^{a_{1}}(u)=\frac{1}{\hat{A}(1)} \int_{-1}^{+1} \frac{1}{a_{1}\left(x_{1}\right)} u\left(x_{1},+1\right) \mathrm{d} x_{1} \\
m_{C}^{a_{1}, b_{2}}(u)=\frac{1}{\hat{A}(1) \hat{B}(1)} \int_{-1}^{+1} \int_{-1}^{+1} \frac{1}{a_{1}\left(x_{1}\right) b_{2}\left(x_{2}\right)} u\left(x_{1}, x_{2}\right) \mathrm{d} x_{1} \mathrm{~d} x_{2} .
\end{array}\right.
$$

On pose par définition :

$$
\begin{aligned}
& R_{h}=\left\{v \in L^{2}(\Omega): \forall \Omega_{i} \in T_{h}, v / \Omega_{i} \in P\left(\Omega_{i}\right)\right\}, \text { et } I=\left\{\left(L, b_{2}\right),\left(R, b_{2}\right),\left(D, a_{1}\right),\left(U, a_{1}\right),\left(C, a_{1} b_{2}\right)\right\} \\
& V_{h}=\left\{v \in R_{h}, m_{E}^{g^{\prime}}(v) \text { est continu à travers les interfaces des } \Omega_{i} \forall\left(E, g^{\prime}\right) \in I \text { et } m_{E}^{g^{\prime}}(v)=0 \text { si } E \subset \partial \Omega\right\} .
\end{aligned}
$$

Soit $P\left(\hat{\Omega}_{i}\right)$ (resp. $\left.\hat{R}_{h}, \hat{V}_{h}\right)$, l'image de $P\left(\Omega_{i}\right)$ (resp. $\hat{R}_{h}, V_{h}$ ) par la transformation (3.4) i.e. $P\left(\hat{\Omega}_{i}\right)=\left\{1, t_{1}\right.$, $\left.t_{2}, t_{1}^{2}, t_{2}^{2}\right\}$,

$$
\begin{aligned}
\hat{R}_{h} & =\left\{\hat{v} \in L^{2}(\hat{\Omega}): \forall \hat{\Omega}_{i}, \hat{v}_{/ \hat{\Omega}_{i}} \in P\left(\hat{\Omega}_{i}\right)\right\} \\
\hat{V}_{h} & =\left\{\hat{v} \in \hat{R}_{h}, m_{E}^{g^{\prime}}(\hat{v}) \text { est continu à travers les interfaces des } \hat{\Omega}_{i} \forall\left(E, g^{\prime}\right) \in I \text { et } m_{E}^{g^{\prime}}(\hat{v})=0 \text { si } E \subset \partial \hat{\Omega}\right\} .
\end{aligned}
$$

On remarque que d'après (3.6) qu'il existe $c>0$

$$
\|\hat{v}\|_{h} \leq c\|v\|_{h} ; \quad \forall v \in V_{h} .
$$


On pose ensuite :

$$
\left\{\begin{array}{l}
u_{L}^{b_{2}}\left(x_{1}, x_{2}\right)=-\frac{1}{2}\left(-\frac{6}{\hat{A}(1)^{2}} \hat{A}\left(x_{1}\right)^{2}+\frac{8}{\hat{A}(1)} \hat{A}\left(x_{1}\right)-2\right) \\
u_{R}^{b_{2}}\left(x_{1}, x_{2}\right)=-\frac{1}{2}\left(-\frac{6}{\hat{A}(1)^{2}} \hat{A}\left(x_{1}\right)^{2}-\frac{4}{\hat{A}(1)} \hat{A}\left(x_{1}\right)\right) \\
u_{D}^{a_{1}}\left(x_{1}, x_{2}\right)=-\frac{1}{2}\left(-\frac{6}{\hat{B}(1)^{2}} \hat{B}\left(x_{2}\right)^{2}+\frac{8}{\hat{B}(1)} \hat{B}\left(x_{2}\right)-2\right) \\
u_{U}^{a_{1}}\left(x_{1}, x_{2}\right)=-\frac{1}{2}\left(-\frac{6}{\hat{B}(1)^{2}} \hat{B}\left(x_{2}\right)^{2}-\frac{4}{\hat{B}(1)} \hat{B}\left(x_{2}\right)\right) \\
u_{C}^{a_{1} b_{2}}\left(x_{1}, x_{2}\right)=-\frac{6}{\hat{A}(1)^{2}} \hat{A}\left(x_{1}\right)^{2}-\frac{6}{\hat{B}(1)^{2}} \hat{B}\left(x_{2}\right)^{2}+\frac{6}{\hat{A}(1)} \hat{A}\left(x_{1}\right)+\frac{6}{\hat{B}(1)} \hat{B}\left(x_{2}\right)-1
\end{array}\right.
$$

Avec ces notations on a :

Lemme 2. Si $m_{E}^{g^{\prime}}$ (resp. $\left.u_{F}^{h^{\prime}}\right)$ est l'une des formes linéaires (resp. des fonctions) définies en (4.4) (resp. en (4.5)), alors on $a$ :

$$
m_{E}^{g^{\prime}}\left(u_{F}^{h}\right)=\delta_{\left(E, g^{\prime}\right)\left(F, h^{\prime}\right)}= \begin{cases}1 & \text { si }\left(E, g^{\prime}\right)=\left(F, h^{\prime}\right) \\ 0 & \text { sinon }\end{cases}
$$

pour tout couple $\left(\left(E, g^{\prime}\right),\left(F, h^{\prime}\right)\right) \in I^{2}$.

En effet, dans le plan $\left(t_{1}, t_{2}\right)$ les fonctions $u_{L}^{b_{2}}, u_{R}^{b_{2}}, u_{D}^{a_{1}}, u_{U}^{a_{1}}$ et $u_{C}^{a_{1} b_{2}}$ peuvent prendre respectivement les formes suivantes :

$$
\left\{\begin{aligned}
u_{L}^{b_{2}} & =-\frac{1}{2}\left(t_{1}-\frac{1}{2}\left(3 t_{1}^{2}-1\right)\right)=-\frac{1}{2}\left(p_{1}\left(t_{1}\right)-p_{2}\left(t_{1}\right)\right) \\
u_{R}^{b_{2}} & =\frac{1}{2}\left(t_{1}+\frac{1}{2}\left(3 t_{1}^{2}-1\right)\right)=\frac{1}{2}\left(p_{1}\left(t_{1}\right)+p_{2}\left(t_{1}\right)\right) \\
u_{D}^{a_{1}} & =\frac{1}{2}\left(t_{2}-\frac{1}{2}\left(3 t_{2}^{2}-1\right)\right)=\frac{1}{2}\left(p_{1}\left(t_{2}\right)+p_{2}\left(t_{2}\right)\right) \\
u_{U}^{a_{1}} & =-\frac{1}{2}\left(t_{2}-\frac{1}{2}\left(3 t_{2}^{2}-1\right)\right)=-\frac{1}{2}\left(p_{1}\left(t_{3}\right)-p_{2}\left(t_{2}\right)\right) \\
u_{C}^{a_{1} b_{2}} & =1-\frac{1}{2}\left(3 t_{1}^{2}-1\right)-\frac{1}{2}\left(3 t_{2}^{2}-1\right)=1-p_{2}\left(t_{1}\right)-p_{2}\left(t_{2}\right)
\end{aligned}\right.
$$

où les $p_{i}$ sont les polynômes de Legendre. On a donc :

$$
m_{L}^{b_{2}}\left(u_{L}^{b_{2}}\right)=\frac{1}{\hat{B}(1)} \int_{-1}^{1} u_{L}^{b_{2}}\left(-1, x_{2}\right) \frac{1}{b_{2}\left(x_{2}\right)} \mathrm{d} x_{2}=\frac{1}{2} \int_{-1}^{1} \hat{u}_{L}^{b_{2}}\left(-1, t_{2}\right) \mathrm{d} t_{2} \quad(\operatorname{par}(3.4))
$$

avec

$$
u_{L}^{b_{2}}\left(t_{1}, t_{2}\right)=-\frac{1}{2}\left(p_{1}\left(t_{1}\right)-p_{2}\left(t_{2}\right)\right)
$$

Donc d'après la théorie des méthodes nodales standards on a :

$$
m_{L}^{b_{2}}\left(u_{L}^{b_{2}}\right)=m_{L}\left(u_{L}\right)=1
$$


De même on obtient :

$$
\begin{aligned}
& m_{L}^{b_{2}}\left(u_{R}^{b_{2}}\right)=\frac{1}{\hat{B}(1)} \int_{-1}^{1} u_{R}^{b_{2}}\left(1, x_{2}\right) \frac{1}{b_{2}\left(x_{2}\right)} \mathrm{d} x_{2}=\frac{1}{2} \int_{-1}^{1} \hat{u}_{R}^{b_{2}}\left(1, t_{2}\right) \mathrm{d} t_{2}=m_{L}\left(u_{R}\right)=0 . \\
& m_{L}^{b_{2}}\left(u_{D}^{a_{1}}\right)=\frac{1}{\hat{B}(1)} \int_{-1}^{1} u_{D}^{a_{1}}\left(-1, x_{2}\right) \frac{1}{b_{2}\left(x_{2}\right)} \mathrm{d} x_{2}=\frac{1}{2} \int_{-1}^{1} \frac{1}{2}\left(p_{1}\left(t_{2}\right)+p_{2}\left(t_{2}\right)\right) \mathrm{d} t_{2}=m_{L}\left(u_{D}\right)=0 . \\
& m_{L}^{b_{2}}\left(u_{U}^{a_{1}}\right)=\frac{1}{4} \int_{-1}^{1} \int_{-1}^{1}\left(1-p_{2}\left(t_{1}\right)-p_{2}\left(t_{2}\right)\right) \mathrm{d} t_{1} \mathrm{~d} t_{2}=m_{L}\left(u_{C}\right)=0 .
\end{aligned}
$$

L'idée donc est d'appliquer le changement de variable (3.4) afin d'écrire les $u_{E}^{g^{\prime}}\left(\left(E, g^{\prime}\right) \in I\right)$ en fonction des polynômes de Legendre dans le plan $\left(t_{1}, t_{2}\right)$ et ensuite d'appliquer les résultats d'unisolvance dans le cas des méthodes nodales standards.

Soit donc $D$ l'ensemble défini en (4.3) et $P\left(\Omega_{i}\right)$ l'espace vectoriel défini en (4.2) on a alors:

Lemme 3. D est P-unisolvant.

En effet, on a $\operatorname{card} D=\operatorname{dim} P=5$, et on prend $u_{C}^{a_{1} b_{2}}$ (resp. $\left.u_{U}^{a_{1}}, u_{L}^{b_{2}}, u_{R}^{b_{2}}, u_{D}^{a_{1}}\right)$ comme fonctions de bases relatives a $m_{C}^{a_{1} b_{2}}\left(\right.$ resp. $\left.m_{U}^{a_{1}}, m_{L}^{b_{2}}, m_{R}^{b_{2}}, m_{D}^{a_{1}}\right)$, le lemme précédent permet de conclure.

La méthode nodale mathématique consiste à considérer la formulation variationnelle (3.1) du problème (1.1) sur l'espace $H_{0}^{1}(\Omega)$, qui va être approché par une suite de sous espaces vectoriels $V_{h}$ (avec $0<h \leq 1$ ) de dimensions finies telles que :

$$
\begin{aligned}
V_{h}=\{ & v \in L^{2}(\Omega): \forall \Omega \in T_{h}, v / \Omega_{i} \in P\left(\Omega_{i}\right), \\
& \left.m_{E}^{g^{\prime}}(v) \text { est continu à travers les interfaces des } \Omega_{i} \forall\left(E, g^{\prime}\right) \in I \text { et } m_{E}^{g^{\prime}}(v)=0 \text { si } E \subset \partial \Omega\right\} .
\end{aligned}
$$

Il est clair que $V_{h} \not \subset H_{0}^{1}(\Omega)$ donc la méthode d'approximation sera non-conforme, ce qui va exiger les rectifications suivantes : la forme bilinéaire $a(.,$.$) définie en (3.2) sera remplacée par :$

$$
a_{h}\left(u_{h}, v_{h}\right)=\sum_{\Omega_{i} \in T_{h}} \int_{\Omega_{i}} \sum_{k=1}^{2} a_{k}\left(x_{1}\right) b_{k}\left(x_{2}\right) \frac{\partial u_{h}}{\partial x_{k}} \frac{\partial v_{h}}{\partial x_{k}}+g\left(x_{1}, x_{2}\right) u_{h} v_{h} \mathrm{~d} x, \quad \forall u_{h}, v_{h} \in V_{h}
$$

sans changer le second membre (3.2) qui est $f(v)$ car $V_{h} \subset L^{2}(\Omega)$, et on pose

$$
\left\|u_{h}\right\|_{h}^{2}=\left[\sum_{\Omega_{i} \in T_{h}} \int_{\Omega_{i}}\left|\nabla u_{h}\right|^{2} d x\right]^{2}, \forall u_{h} \in V_{h}
$$

Lemme 4. Si $V_{h}$ est l'espace défini en (4.6) et $\|\cdot\|_{h}$ comme définie en (4.7) alors $\|\cdot\|_{h}$ est une norme sur $V_{h}$.

En effet, il est clair qu'il s'agit d' une semi-norme sur $V_{h}$ soit donc $u_{h} \in V_{h}$ tel que : $\left\|u_{h}\right\|_{h}=0$ i.e. $\sum_{\Omega_{i} \in T_{h}} \int_{\Omega_{i}}\left|\nabla u_{h}\right|^{2} \mathrm{~d} x=0$ c'est-à-dire $u_{h} / \Omega_{i}=c_{i}$ car $\Omega_{i}$ est connexe ; or la continuité des moments avec poids sur les interfaces des $\Omega_{i}$ implique que sur les frontières verticales (FV) des $\Omega_{i}$ (voir Fig. 3) on a :

$$
\left(c_{i}-c_{j}\right) \int_{-1}^{+1} \frac{1}{a_{1}(t)} \mathrm{d} t=0 \longrightarrow c_{i}=c_{j}
$$

et sur les frontières horizontales (FH) des $\Omega_{i}$ on a :

$$
\left(c_{i}-c_{m}\right) \int_{-1}^{+1} \frac{1}{b_{2}(t)} \mathrm{d} t=0 \longrightarrow c_{i}=c_{m}
$$

c'est-à-dire les constantes sont toutes égales a une constante $c$, et puisque $u_{h}$ admet des moments nuls sur les $\partial \Omega$ on a $c=0$ par suite $u_{h}=0$. 


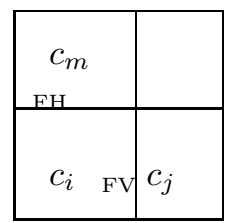

FiguRe 3.

De même on peut munir $V_{h}$ de la norme suivante :

$$
\|v\|_{1, h}^{2}=\sum_{\Omega_{i} \in T_{h}}\|v\|_{1, \Omega_{i}}^{2}
$$

On remarque que la forme bilinéaire $a_{h}(.,$.$) vérifie les conditions suivantes :$

$$
\left\{\begin{array}{l}
\left|a_{h}\left(u_{h}, v_{h}\right)\right| \leq c_{2}\left\|u_{h}\right\|_{h}\left\|v_{h}\right\|_{h} \\
c_{1}\left\|u_{h}\right\|_{h}^{2} \leq a_{h}\left(u_{h}, u_{h}\right), \forall u_{h}, v_{h} \in V_{h} \\
c_{1}, c_{2} \text { deux constantes definies au paragraphe } 1
\end{array}\right.
$$

On va étendre le domaine de définition de la norme $\|\cdot\|_{h}$ et la forme bilinéaire $a_{h}(.,$.$) à l'espace \left(V_{h}+H_{0}^{1}(\Omega)\right)$ tout en respectant les notations suivantes :

$$
a_{h}(u, v)=a(u, v), \quad\|u\|_{h}=\|u\|_{1, \Omega}, \forall u, v \in H_{0}^{1}(\Omega)
$$

D'après (4.9) il existe une solution et une seule du problème suivant :

$$
\left\{\begin{array}{l}
u_{h} \in V_{h} \\
a_{h}\left(u_{h}, v_{h}\right)=f\left(v_{h}\right), \quad \forall v_{h} \in V_{h}
\end{array}\right.
$$

Étant donné $u \in H^{L}(\Omega)$, on définit $\Pi_{\Omega_{i}}(u)$, l'interpolant local de $u$ sur $\Omega_{i}$ par :

$$
\left\{\begin{array}{l}
\Pi_{\Omega_{i}}(u) \in P\left(\Omega_{i}\right) \\
m_{E}^{g^{\prime}}(u)=m_{E}^{g^{\prime}}\left(\Pi_{\Omega_{i}}(u)\right), \quad \forall\left(E, g^{\prime}\right) \in I
\end{array}\right.
$$

On désigne par $\Pi_{\Omega}(u)$ l'interpolant global de $u$ défini sur $\Omega \operatorname{par}\left(\Pi_{\Omega}(u)\right) / \Omega_{i}=\Pi_{\Omega_{i}}(u)$ et on pose $\Pi_{\Omega}(u)=\Pi u$ pour alléger l'écriture, on a le résultat suivant:

Lemme 5. Si u est la solution de (1.1) et $\Pi u$ est le $P(\Omega)$-interpolant de $u$ dans $V_{h}$ alors on a :

$$
\|u-\Pi u\|_{h} \leq c^{\prime} h\|f\|_{0, \Omega}
$$

où $c^{\prime}=c^{\prime}\left(c_{1}, c_{2}, \sigma\right)$. 
En effet,

$$
\begin{aligned}
\|u-\Pi u\|_{h}^{2}= & \sum_{\Omega_{i} \in T_{h}} \int_{\Omega_{i}}\left|\nabla\left(u-\Pi_{\Omega_{i}} u\right)\right|^{2} \mathrm{~d} x \\
= & \sum_{\hat{\Omega}_{i} \in \hat{T}_{h}} \int_{\hat{\Omega}_{i}} \frac{\hat{b}_{2}\left(t_{2}\right)}{\hat{a}_{1}\left(t_{1}\right)}\left|\frac{\partial}{\partial t_{1}}\left(\hat{u}-\Pi_{\hat{\Omega}_{i}} \hat{u}\right)\right|^{2} \mathrm{~d} t \\
& +\sum_{\hat{\Omega}_{i} \in \hat{T}_{h}} \int_{\hat{\Omega}_{i}} \frac{\hat{a}_{1}\left(t_{1}\right)}{\hat{b}_{1}\left(t_{2}\right)}\left|\frac{\partial}{\partial t_{2}}\left(\hat{u}-\Pi_{\hat{\Omega}_{i}} \hat{u}\right)\right|^{2} \mathrm{~d} t \quad \text { d'après (3.4). }
\end{aligned}
$$

Or $u \in H^{L}(\Omega)$ donc $\hat{u} \in H^{2}(\hat{\Omega})$ et $\Pi_{\hat{\Omega}_{i}}(\hat{u}) \in\left\langle 1, t_{1}, t_{2}, t_{1}^{2}, t_{2}^{2}\right\rangle$, donc d'après la théorie d'interpolation on a :

$$
\begin{aligned}
\|u-\Pi u\|_{h}^{2} & \leq c \sum_{\hat{\Omega}_{i} \in \hat{T}_{h}} \int_{\hat{\Omega}_{i}}\left|\nabla\left(\hat{u}-\Pi_{\hat{\Omega}_{i}} \hat{u}\right)\right|^{2} \mathrm{~d} x \leq c \sum_{\hat{\Omega}_{i} \in \hat{T}_{h}}\left|\hat{u}-\Pi_{\hat{\Omega}_{i}} \hat{u}\right|_{1, \hat{\Omega}_{i}}^{2} \\
& \leq c \sum_{\hat{\Omega}_{i} \in T_{h}} \hat{h}_{i}^{2} \sigma^{2}|\hat{u}|_{2, \hat{\Omega}_{i}}^{2} \\
& \left.\leq c h^{2}\|f\|_{0, \Omega}^{2}, \quad \text { (d'après }(2.2,3.6,3.9,4.1)\right) .
\end{aligned}
$$

Par suite :

$$
\|u-\Pi u\|_{h} \leq c^{\prime} h\|f\|_{0, \Omega} .
$$

Lemme 6. Si u est la solution du problème (1.1) alors on a :

$$
\sup _{w_{h} \in V_{h}} \frac{\left|a_{h}\left(u, w_{h}\right)-f\left(w_{h}\right)\right|}{\left\|w_{h}\right\|_{h}} \leq \operatorname{ch}\|f\|_{0, \Omega} .
$$

En effet, soit $w_{h} \in V_{h}$ on a :

$$
a_{h}\left(u, w_{h}\right)-f\left(w_{h}\right)=\sum_{\Omega_{i} \in T_{h}} \int_{\Omega_{i}}\left(a_{1} b_{1} u_{x_{1}} w_{h x_{1}}+a_{2} b_{2} u_{x_{2}} w_{h x_{2}}-f w_{h}\right) \mathrm{d} x
$$

avec $a_{i}\left(\right.$ resp. $\left.b_{i}\right)$ sont $a_{i}\left(x_{1}\right)\left(\right.$ resp. $\left.b_{i}\left(x_{2}\right)\right)$ et $u_{x_{i}}\left(\right.$ resp. $\left.w_{h x_{i}}\right)=\partial u / \partial x_{i}\left(\right.$ resp. $\left.\partial w_{h} / \partial x_{i}\right)$.

D'après la transformation (3.4) on a :

$$
\begin{aligned}
a_{h}\left(u, w_{h}\right)-f\left(w_{h}\right)= & \sum_{\hat{\Omega}_{i} \in T_{h}} \int_{\hat{\Omega}_{i}}\left(\hat{b}_{1} \hat{b}_{2} \hat{u}_{t 1} \hat{w}_{h t_{1}}+\hat{a}_{1} \hat{a}_{2} \hat{u}_{t 2} \hat{w}_{h t_{2}}+\hat{g} \hat{u} \hat{w}_{h} \hat{a}_{1} \hat{b}_{2}-\hat{f} \hat{w}_{h} \hat{a}_{1} \hat{b}_{2}\right) \mathrm{d} t \\
= & \sum_{\hat{\Omega}_{i} \in T_{h}} \int_{\hat{\Omega}_{i}}\left(\hat{b}_{1} \hat{b}_{2} \hat{u}_{t 1} \hat{w}_{h t_{1}}+\hat{a}_{1} \hat{a}_{2} \hat{u}_{t 2} \hat{w}_{h t_{2}}+\hat{g} \hat{u} \hat{w}_{h} \hat{a}_{1} \hat{b}_{2}\right) \mathrm{d} t \quad \quad \quad \text { voir } \\
& +\sum_{\hat{\Omega}_{i} \in T_{h}} \int_{\hat{\Omega}_{i}}\left(\hat{b}_{1} \hat{b}_{2} \hat{u}_{t_{1} t_{1}} \hat{w}_{h}+\hat{a}_{1} \hat{a}_{2} \hat{u}_{t 2} \hat{w}_{h t_{2} t_{2}}-\hat{g} \hat{u} \hat{w}_{h} \hat{a}_{1} \hat{b}_{2}\right) \mathrm{d} t \\
= & \sum_{\hat{\Omega}_{i} \in T_{h}} \int_{\hat{\Omega}_{i}}\left(\hat{b}_{1} \hat{b}_{2} \hat{u}_{t 1} \hat{n}_{1}+\hat{a}_{1} \hat{a}_{2} \hat{u}_{t 2} \hat{n}_{2}\right) \hat{w}_{h} \mathrm{~d} \hat{s} \quad \text { (formule de Green) }
\end{aligned}
$$


où d $\hat{s}$ est l'élément de surface de $\partial \hat{\Omega}_{i}$ et $\hat{n}=\left(n_{1}, n_{2}\right)$ est la normale extérieure à $\partial \hat{\Omega}_{i}$ donc (4.12) est égale à :

avec

$$
\sum_{\hat{\Omega}_{i} \in \hat{T}_{h}} \int_{\partial \hat{\Omega}_{i}}(\hat{A} \nabla \hat{u} . \vec{n}) \hat{w}_{h} \mathrm{~d} \hat{s},
$$

$$
\hat{A}=\left[\begin{array}{cc}
\hat{b}_{1} \hat{b}_{2} & 0 \\
0 & \hat{a}_{1} \hat{a}_{2}
\end{array}\right] .
$$

Les éléments de $\hat{A}$ sont dans $\left.L^{\infty}(I) \subset L^{2}(I), I=\right]-1,+1[$ donc pour tout $\varepsilon>0$ il existe une matrice diagonale $\hat{B}$ dont les éléments sont des fonctions continues telles que :

$$
\|\hat{A}-\hat{B}\|_{0, \partial \hat{\Omega}} \leq \varepsilon, \text { avec }\|\hat{A}-\hat{B}\|_{0, \partial \hat{\Omega}}^{2}=\sum_{i=1}^{2}\left\|\hat{a}_{i i}-\hat{b}_{i i}\right\|_{0, \partial \hat{\Omega}}^{2}
$$

Donc

$$
\begin{aligned}
a\left(u, w_{h}\right)-f\left(w_{h}\right) & =\sum_{\hat{\Omega}_{i} \in \hat{T}_{h}}\left(\int_{\partial \hat{\Omega}_{i}}((\hat{A}-\hat{B}) \nabla \hat{u} \cdot \vec{n}) \hat{w}_{h} \mathrm{~d} \hat{s}+\sum_{\hat{\Omega}_{i} \in T_{h}} \int_{\partial \hat{\Omega}_{i}} \hat{B} \nabla \hat{u} \cdot \vec{n} \hat{w}_{h} \mathrm{~d} \hat{s}\right) \\
& \leq \sum_{\hat{\Omega}_{i} \in T_{h}}\|\hat{A}-\hat{B}\|_{0, \partial \hat{\Omega}}\|\nabla \hat{u}\|_{0, \partial \hat{\Omega}_{i}}\left\|\hat{w}_{h}\right\|_{0, \partial \hat{\Omega}_{i}}+\int_{\partial \hat{\Omega}_{i}} \hat{B} \nabla \hat{u} \cdot \vec{n} \hat{w}_{h} \mathrm{~d} \hat{s} \\
& \leq c \varepsilon\|\hat{u}\|_{2, \hat{\Omega}}\left\|\hat{w}_{h}\right\|_{h}+\int_{\partial \hat{\Omega}_{i}} \hat{B} \nabla \hat{u} \cdot \vec{n} \hat{w}_{h} \mathrm{~d} \hat{s}, \quad c=c\left(c_{1}, c_{2}, \sigma\right) .
\end{aligned}
$$

Ceci d'après le théorème de trace appliqué à $\left(\nabla \hat{u}, \hat{w}_{h}\right)$, et la formule $(4.8)$.

Soit $I(\hat{B} \nabla \hat{u} . \vec{n})$ l'interpolant constant de $\hat{B} \nabla \hat{u} . \vec{n}$ sur les $\partial \hat{\Omega}_{i}$, alors $I(\hat{B} \nabla \hat{u} . \vec{n})$ et $\hat{B} \nabla \hat{u} . \vec{n}$ ont la même valeur moyenne sur les $\partial \hat{\Omega}_{i}$, et puisque $\hat{w}_{h}$ admet des moments continus (d'ordre zéro) sur les $\partial \hat{\Omega}_{i}$ on a alors :

$$
a\left(u, w_{h}\right)-f\left(w_{h}\right) \leq c \varepsilon\|f\|_{0, \Omega}\left\|\hat{w}_{h}\right\|_{h}+\sum_{\hat{\Omega}_{i} \in T_{h}} \int_{\partial \hat{\Omega}_{i}}(\hat{B} \nabla \hat{u} \cdot \vec{n}-I(\hat{B} \nabla \hat{u} . \vec{n})) \hat{w}_{h} \mathrm{~d} \hat{s} .
$$

Or la fonctionnelle

$$
\hat{w}_{h} \longrightarrow \int_{\partial \hat{\Omega}_{i}}(\hat{B} \nabla \hat{u} \cdot \vec{n}-I(\hat{B} \nabla \hat{u} \cdot \vec{n})) \hat{w}_{h} \mathrm{~d} \hat{s}
$$

s'annule pour $\hat{w}_{h}=c$, ainsi par application du théorème de Bramble-Hilbert [4], la théorie d'interpolation et les formules $(3.6,3.9)$ on a :

$$
a\left(u, w_{h}\right)-f\left(w_{h}\right) \leq c \varepsilon\|f\|_{0, \Omega}\left\|w_{h}\right\|_{h}+c^{\prime} h\|f\|_{0, \Omega}\left\|w_{h}\right\|_{h} .
$$

Donc pour $\varepsilon=\varepsilon(h)=h$ et d'après $(3.6,3.7,3.10)$ on a :

$$
\frac{a\left(u, w_{h}\right)-f\left(w_{h}\right)}{\left\|w_{h}\right\|_{h}} \leq c^{\prime \prime} h\|f\|_{0, \Omega} .
$$

Ceci étant, le deuxième lemme de Strang ([5], p. 212) et les deux lemmes précédents donnent:

Théorème 4. Si $u_{h}$ est la solution du problème discret (4.10) on $a$ :

$$
\left\|u-u_{h}\right\|_{h} \leq c\left(\inf _{v_{h} \in V_{h}}\left\|u-v_{h}\right\|_{h}+\sup _{w_{h} \in V_{h}} \frac{\left|a_{h}\left(u, w_{h}\right)-f\left(w_{h}\right)\right|}{\left\|w_{h}\right\|_{h}}\right) \leq \bar{c} h
$$

où $\bar{c}=\bar{c}\left(c_{1}, c_{2}, \sigma, f\right)$. 


\section{Majoration de l'erreur $\left\|u_{-} u_{h}\right\|_{0, \Omega}$}

Le problème adjoint du problème (1.1) est le suivant :

$$
\left\{\begin{array}{l}
q_{g} \in H_{0}^{1}(\Omega) \\
a\left(v, q_{g}\right)=(g, v), \forall v \in H_{0}^{1}(\Omega), g \in L^{2}(\Omega)
\end{array}\right.
$$

La forme bilinéaire $a(.,$.$) étant symétrique donc le problème (4.13), vu les conditions de Lax-Milgram prises sur$ $a(.,$.$) admet une solution unique q_{g} \in H_{0}^{1}(\Omega)$ le même procédé du paragraphe 3 montre que $q_{g} \in H_{0}^{1}(\Omega) \cap H^{L}(\Omega)$, et si on désigne par $\left(q_{g}\right)_{h}$ l'équivalent de $u_{h}$ pour le problème (1.1) on a :

$$
\left\{\begin{array}{l}
\left\|q_{g-}\left(q_{g}\right)_{h}\right\|_{h} \leq c h \text { et } \\
\sup _{q_{g} \in V_{h}} \frac{\left|a\left(q_{g},\left(q_{g}\right)_{h}\right)_{-}\left(g, q_{g}\right)\right|}{\left\|\left(q_{g}\right)_{h}\right\|_{h}} \leq c h .
\end{array}\right.
$$

Finalement le lemme d'Aubin-Nitsche $[11,12]$ nous permet d'avoir :

$$
\left\|u_{-} u_{h}\right\|_{0, \Omega} \leq c h^{2} .
$$

\section{RÉSUltats NUMÉRIQUES}

Notre domaine de travail sera toujours $\Omega=]-1,+1\left[2\right.$, on désigne par $\varepsilon$ l'erreur dans la norme $L^{2}$ i.e. $\varepsilon=\left\|u_{-} u_{h}\right\|_{0, \Omega}$ et $\alpha$ l'ordre de convergence $\left(h^{\alpha}\right)$.

\subsection{Cas des coefficients constants par morceaux}

On considère la fonction $u\left(x_{1}, x_{2}\right)=\left(1-x_{1}^{2}\right)\left(1-x_{2}^{2}\right)$ solution de $-\Delta u+u=f$ avec :

$$
f\left(x_{1}, x_{2}\right)=5-3\left(x_{1}^{2}+x_{2}^{2}\right)+x_{1}^{2} x_{2}^{2} .
$$

On obtient les résultats suivants :

TABleau 1. Cas des coefficients constants par morceaux.

\begin{tabular}{cll}
\hline Triangulation & \multicolumn{1}{c}{$\varepsilon$} & $\alpha$ \\
\hline $2 \times 2$ & 1,631 & $-\overline{1,699}$ \\
$4 \times 4$ & 0,5649 & 1,889 \\
$8 \times 8$ & 0,1582 & 2,001 \\
$16 \times 16$ & 0,03947 & \\
\hline
\end{tabular}

\subsection{Cas des coefficients variables}

Dans ce cas $\Omega$ va être partagé en quatre zones selon les droites $x_{1}=1 / 3$, et $x_{2}=1 / 6$, les coefficients $a_{i}$ et $b_{i}$ sont pris tels que :

$$
\begin{gathered}
a_{1}(x)=\left\{\begin{array}{ll}
\mathrm{e}^{x_{1}} & x_{1} \geq 1 / 3 \\
10\left(x_{1}+5\right) & x_{1} \leq 1 / 3
\end{array} \quad b_{1}\left(x_{2}\right)= \begin{cases}1 /\left(x_{2}+10\right) & x_{2} \geq 1 / 6 \\
\mathrm{e}^{-x_{2}} & x_{2} \leq 1 / 6\end{cases} \right. \\
a_{2}\left(x_{1}\right)=\left\{\begin{array}{ll}
\mathrm{e}^{-x_{1}} & x_{1} \geq 1 / 3 \\
\left(x_{1}+5\right)^{-1} 1 / 10 & x_{1} \leq 1 / 3
\end{array} \quad b_{2}\left(x_{2}\right)= \begin{cases}x_{2}+10 & x_{2} \geq 1 / 6 \\
\mathrm{e}^{x_{2}} & x_{2} \leq 1 / 6 .\end{cases} \right.
\end{gathered}
$$


TABleau 2. Cas des coefficients variables.

\begin{tabular}{ccc}
\hline Triangulation & $\varepsilon$ & $\alpha$ \\
\hline $2 \times 2$ & 4,225 & $-\overline{1,557}$ \\
$4 \times 4$ & 1,652 & 1,814 \\
$8 \times 8$ & 0,501 & 1,975 \\
$16 \times 16$ & 0,286 & 2,011 \\
$32 \times 32$ & 0,142 & \\
\hline
\end{tabular}

Avec ces coefficients, on considère le problème (1.1) avec $g=0$, et le second membre :

$$
f\left(x_{1}, x_{2}\right)=\left\{\begin{array}{lll}
+2 \mathrm{e}^{x_{1}}\left(x_{2}-1\right) /\left(x_{2}+10\right)-2 \mathrm{e}^{-x_{1}}\left(1-x_{1}\right) & x_{1} \geq 1 / 3, & x_{2} \geq 1 / 6 \\
-10\left(x_{2}-1\right) /\left(x_{2}+10\right)-\left(x_{1}+1\right) /\left(10 x_{1}+50\right) & x_{1} \leq 1 / 3, & x_{2} \geq 1 / 6 \\
50 \mathrm{e}^{-x_{2}}\left(x_{2}+1\right) / 7+\mathrm{e}^{x_{2}}\left(x_{1}+1\right) /\left(14 x_{1}+70\right) & x_{1} \leq 1 / 3, & x_{2} \leq 1 / 6 \\
10 \mathrm{e}^{-x_{2}} \mathrm{e}^{x_{1}}\left(x_{2}+1\right)+10 \mathrm{e}^{-x_{1}} \mathrm{e}^{x_{2}}\left(1-x_{1}\right) / 7 & x_{1} \geq 1 / 3, & x_{2} \leq 1 / 6
\end{array}\right.
$$

La solution exacte s'écrit :

$$
u\left(x_{1}, x_{2}\right)=\left\{\begin{array}{lll}
2\left(1-x_{1}\right)\left(x_{2}-1\right) & x_{1} \geq 1 / 3, & x_{2} \geq 1 / 6 \\
\left(x_{1}+1\right)\left(x_{2}-1\right) & x_{1} \leq 1 / 3, & x_{2} \geq 1 / 6 \\
-5\left(x_{1}+1\right)\left(x_{2}+1\right) / 7 & x_{1} \leq 1 / 3, & x_{2} \leq 1 / 6 \\
-10\left(1-x_{1}\right)\left(x_{2}+1\right) / 7 & x_{1} \geq 1 / 3, & x_{2} \leq 1 / 6
\end{array}\right.
$$

Résultats obtenus : voir tableau 2.

\section{Conclusion}

Quand les coefficients $a_{i}, b_{i}$ sont constants on a $P\left(\Omega_{i}\right)=\left\langle 1, x_{1}, x_{1}^{2}, x_{2}, x_{2}^{2}\right\rangle$ donc il est clair que cette méthode généralise la méthode nodale standard d'ordre zéro, d'autre part la triangulation adoptée est libre et peut être éventuellement irrégulière, en plus elle n'exige pas que les bords $\partial \Omega_{i}$ coïncident avec les discontinuités des termes $a_{i}, b_{i}$ et n'oblige pas la solution d'être dans $H^{2}(\Omega)$ pour avoir une approximation d'erreur d'ordre $O\left(h^{2}\right)$ dans $L^{2}(\Omega)$.

\section{REFERENCES}

[1] I. Babuska, G. Caloz et J.E. Osborn, Special F.E.M. for a class of second order elliptic problems with rough coefficients. SIAM J. Numer. Anal. 31 (1994) 945-981.

[2] I. Babuska et J.E. Osborn, F.E.M. for the solution of problem with rough imput data. Lect. Notes Math., Springer-Verlag 1121 (1985) 1-18.

[3] S.N. Bernstein, Sur la généralisation du problème de Dirichlet. Math. Annal. 69 (1910) 82-136.

[4] J.H. Bramble et S.R. Hilbert, Estimation of linear functionals on Sobolev spaces with application to Fourier transforms and spline interpolation. SIAM J. Numer. Anal. 7 (1970) 113-124.

[5] P.G. Ciarlet, Handbook of numerical analysis, F.E.M, Part II. North-Holland (1991).

[6] J.P. Hennart, On numerical analysis of analytical nodal methods, Numer. Methods Partial Differential Equations 4 (1986) 233-254.

[7] J.P. Hennart, A general family of nodal shemes. SIAM J. Sci. Statist. Comput. 7 (1986) 264-287.

[8] J.P. Hennart and E.H. Mund, Singularities in the F.E. approximation of two-dimensional diffusion problems. Nucl. Sci. Engng. 62 (1977) 55-68.

[9] M.A. Moussaoui et A. Ziani, Sur l'approximation des solutions de certains types d'E.D. ou d'E.D.P. à coefficients non réguliers. Anales de l'Enit 4 (1990) 9-35.

[10] O.A. Ladyzhenskaya et N.N. Oral'tseva, Linear and quasilinear equations. Academic Press, New York (1968).

[11] R.D. Lawrence, Progress in nodal methods for the solution of the neutron diffusion and transport equation. Prog. Nucl. Energy 17 (1986) 271-301.

[12] P. Lesaint, On the convergence of Wilson's nonconforming element for solving the elastic problem. Comput. Methods Appl. Mech. Engrg. 7 (1976) 1-16. 